\title{
Global Perspective of Developmental Care - Cyprus
}

Pani Pantelides, PT

Consultant Neonatal Physiotherapist and Early Intervention Specialist

Senior Faculty of the Family and Infant Neurodevelopmental Education (FINE)

NIDCAP Professional, NDT

C yprus is a small island situated in the south eastern Mediterranean. The Republic of Cyprus was founded in 1960, after decades of colonial British rule and it joined the European Union in 2004 and the Eurozone in 2008. Since the Turkish invasion in 1974 the island has been halfoccupied and the following article refers only to the situation in the area over which the Government of the Republic of Cyprus exercises effective control and where the Acquis Communautaire of the European Union is fully implemented.

There are two NICUs in this part of Cyprus: A Level III NICU, in the Archbishop Makarios public hospital in the capital Nicosia where 800-900 newborns are treated per year, and a Level IIB, in the general public hospital in Limassol that treats around 350 newborns per year. According to the most recent official data, the Rate of Preterm Births in Cyprus is 12\%, the Neonatal Mortality Rate is $1.9 / 1000$ and the Infant Mortality Rate is $2 \cdot 1 / 1000$. Annual births are between 8,000 and 9,000. Due to lack of community support services, babies spend longer in the hospital than they might where there are community nurses.

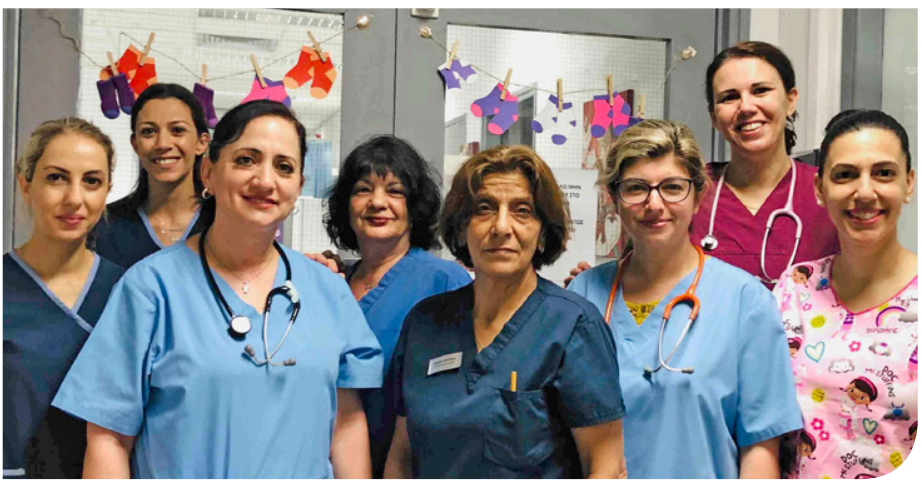

Limmasol team - left to right: Elena Vasiliadou, Ifigenia Smila, Maria Zeniou (NIDCAP Professional), Skevi Andorka, Christoulla Vasiliou, Eleftheria Konstantoulaki, Stalo Ioannou, Matina Lamari.

The NICU in Nicosia was founded in 1984, by Dr Andreas Hadjidemetriou, the first Cypriot neonatologist. With dedication and maximum personal effort he created the very first NICU in Cyprus from scratch. His vision was not only to reduce the mortality of sick and premature infants, but also to ensure the best quality of life for these infants. For this reason, he actively supported the implementation of developmental care and with his zeal and perseverance he inspired his successors to do the same. I had the privilege to work under Dr Hadjidemetriou's mentoring during my working term in the Archbishop Makarios Hospital (2007-2013), when we laid the foundation for developmental care. We are still collaborating today, on a voluntary basis, through the parents' organisation "Thermokoititha Agapis" ("Incubator of Love"), despite his retirement and my shift to the private sector.

My fulltime collaboration with the Nicosia Unit, as Developmental Care Leader, began in 2007, since the gradual training of all staff on the implementation of developmental care, became one of Dr Hadjidemetriou's priorities. On the occasion of my own training to become NIDCAP Professional (2008-2010) and Dr Maria Zeniou's NIDCAP training (2010-2012), the Nicosia Unit initiated close collaboration with Dr Inga Warren, former Director of the UK NIDCAP Centre at St Mary's Hospital in London. Dr Warren has generously been providing consultation to our unit through various baby observation workshops based on the NIDCAP model, for all the staff. Under her valuable guidance in 2009, we managed to create the first protocols of care and in 2010 we began a new institution, the "Parents School", which was about weekly meetings between me and parents, in order to support their communication and interaction with their babies. The "Parents School" evolved gradually and in 2013, a psychologist and a nurse were added.

In 2010, we were pleased to run the first kangaroo care training for members of the staff with Dr Nils Bergman. Since then, we are making consistent efforts to increase the number of babies who enjoy "skin to skin" care.

The turning point in developmental care implementation was in 2012 with the Foundation Course: Individualised Family Centred Developmental Care run by Dr Warren, Ms Bond and myself as an assistant. After that course we decided that it was best to take actions to decentralize developmental care, in order to make more people responsible for preparing strategies, taking actions and initiating staff education.

For this reason, we established a Central (Scientific) Multidisciplinary Developmental Care Committee and a sub-committee of 13 nurses that were responsible for spreading knowledge to the rest of the staff. In order to support staff education in using the new protocols of care we named an intensive care room as the "educational nursery room" in which the staff was expected to practice according to the new protocols. The Educational Nursery Room helped everybody to change their mentality in 


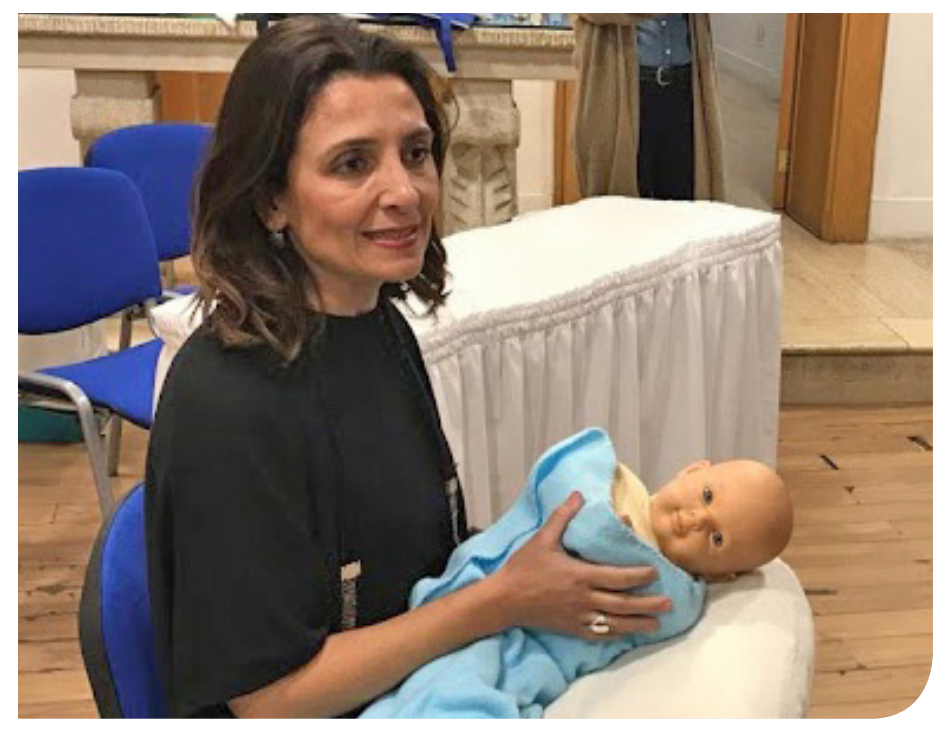

Pani Pantelides - Consultant Neonatal Physiotherapist and Early Intervention Specialist demonstrating positioning using a doll.

caregiving, improved practical skills in developmental care and minimized the gap between training and practical skills.

Unfortunately, due to overcrowding and understaffing of the NICU, (1 nurse for 4-6 newborns in intensive care and 1 nurse for 8-10 newborns in special care) the above measures faded and the standard application of protocols became inconsistent. Finding ways to train our staff was always a big challenge for us and the shortage of nurses inevitably reduces training time.

FINE (Family and Infant Neurodevelopmental Education) was the next big step in family centred care for both NICUs thanks to the Director of the Ministry of Health's Nursing Services, Andreas Xenophontos, who passionately supported FINE training and has put it under his auspices. This course is mostly taught in Greek by myself and Dr Maria Zeniou under the direction of Dr Inga Warren. The goal is for all staff in the NICUs, to follow FINE 1 and for those possible, FINE 2. Until recently, five staff members have completed FINE 2 and three more are in the final stage. We have also been able to work with Dr Warren to introduce FINE 1 and FINE 2 in Greece. Unfortunately, due to the COVID-19 pandemic we had to postpone our second FINE 1 training.

In Nicosia, despite the difficult conditions mentioned above, some basic elements of NIDCAP implementation have been achieved. There are measures taken towards comfortable and developmentally supportive handling, positioning, limitation of noise, light and other environmental stimuli and protection of sleep. Moreover, some first steps have been made for pain management with the use of special pacifiers for premature babies and positioning aids and a protocol to use sucrose or human milk is in development. Sometimes stressful/painful procedures take place during Kangaroo Care, the implementation of which lately has been expanded in the intensive care rooms as well. Parents' interaction and involvement in their baby's care is slowly progressing. Until recently, parents were allowed to be in any room of the NICU for only one hour at noon and one hour in the afternoon. Resistance to parents' participation, partly driven by concerns about overcrowding (the intensive care rooms are extremely cramped), is gradually being overcome with FINE training. As a result, since the beginning of 2020, parents are welcomed for one hour at noon and for two hours in the afternoon in the intensive care rooms and for 12 hours (7:00-19:00) in the special care rooms. Care giving from parents is allowed only in special care and includes only feeding, bathing and kangaroo care. The goal is to enable parents to be in all rooms in the NICU any time on a daily basis and take care of their babies completely. Last but not least, the Archbishop Makarios Hospital in Nicosia is working towards becoming a Baby Friendly Hospital with steps to support breast feeding in the unit and the use of human milk.

The Limassol NICU was upgraded from Level IIA to IIB, in 2018 and is fortunate enough to have a full time NIDCAP Professional, Dr Maria Zeniou, as Developmental Care leader, who in addition to her medical duties, leads the Developmental Care team which includes a FINE 2 trained developmental physiotherapist and members of the nursing staff who have attended the FINE I course. The continuous presence of a specialised physiotherapist is crucial for the newborns' neurodevelopment and the training of the parents for the post NICU period. In addition, all the staff is duly trained in order to control and minimize environmental stimuli.

Remarkable effort has been made in Limassol to implement family centred care. Parents are welcomed 24/7 and they take full care of their babies from the moment they come out of the incubator. They are guided for Kangaroo Care on a daily basis and maternal breastfeeding is encouraged from birth as the Limassol Hospital is close to becoming a Baby Friendly Hospital. Moreover, there is a protocol to use sucrose or human milk during painful procedures and there is always someone available to support and comfort the newborn. All necessary care giving procedures are planned in order not to interrupt newborns' sleep.

Our next big step towards family centred care in Cyprus is the upcoming renovation of the NICU in Nicosia. With this renovation, among other things, a resting area will be created for parents with the possibility of sleeping and preparing simple meals and more bedside space will be available for a kangaroo care chair to fit and for parents to be more involved in their baby's care. In addition, there will be an upgrade of the NICU's sound insulation.

None of the above would have been achieved without the generous support of the parents' organization, "Thermokoititha Agapis" ("Incubator of Love") which organizes and finances all staff training regarding developmental care, provides the two NICUs with developmental care equipment and has taken over, in collaboration with the Ministry of Health, the renovation of the NICU in Nicosia. Last but not least, the full membership of "Thermokoititha Agapis" in EFCNI proved to be crucial and extremely valuable, not only for the implementation in Cyprus of the European Standards of Care for Newborn Health: Standards on Infant and Family Centred Developmental Care (2018), but also for the negotiations with the Ministry of Health and other stakeholders, on the matter. 